\title{
Lifestyle-related determinants of inflammation in adolescence
}

\author{
Julia Wärnberg ${ }^{1,2}$, Esther Nova ${ }^{1}$, Javier Romeo ${ }^{1}$, Luís A. Moreno ${ }^{3}$, Michael Sjöström ${ }^{2}$ and \\ Ascensión Marcos ${ }^{1}$ \\ ${ }^{1}$ Immunonutrition Research Group, Department of Metabolism and Nutrition, Instituto del Frío, Consejo Superior de \\ Investigaciones Cientificas (CSIC), Madrid, Spain \\ ${ }^{2}$ Unit for Preventive Nutrition, Department of Biosciences and Nutrition, Karolinska Institutet, Stockholm, Sweden \\ ${ }^{3}$ E.U. Health Sciences, University of Zaragoza, Zaragoza, Spain
}

Inflammatory processes are involved in the pathogenesis of the most common chronic non-communicable diseases and may also play an important initiating role in their development. Only recently have inflammatory markers been included in epidemiological studies focusing on nutritional status, body composition and physical activity. We are just starting to understand how different lifestyles can determine basal levels of inflammatory biomarkers in early ages. This review aims to summarise what is known about the relationships between lifestyle-related determinants (focusing on overweight, physical activity and dietary habits) and inflammatory markers in apparently healthy young populations. Obesity is the most widely studied determinant. Several large-scale studies have now demonstrated that healthy young subjects with more body fat or higher BMI have moderately higher concentrations of inflammatory markers than their leaner peers, supporting the idea that obesity should be considered as a state of chronic low-grade inflammation. Less data is available to allow us to elucidate how physical activity/fitness or dietary patterns may have a direct effect on inflammation in apparently healthy, disease-free young populations.

Obesity: Exercise: Diet: Acute-phase proteins

The immune system is intimately linked to health disorders such as obesity, type 2 diabetes and cardiovascular disease (CVD) and may also play an important initiating role in the development of these and other chronic diseases. It is of special interest to study these interrelationships in young, disease-free populations, where interaction effects by any ongoing chronic inflammatory pathology that could be present in an adult apparently disease-free population, are minimised. To target groups for special attention or to evaluate what kind of promotion is most effective to prevent chronic diseases, the study of new biomarkers such as mediators of the inflammatory processes is important. Because CVD often has its origins in young age and because several risk factors for CVD track from childhood to adulthood, understanding the distribution and implications of such risk factors among adolescents is of considerable interest for the prevention of future diseases while there is still an opportunity to reverse any damage.

This review aims to summarise published data on the relationships between lifestyle-related determinants (i.e. overweight, physical activity/fitness and dietary habits) and inflammatory markers in apparently healthy young populations.

\section{Inflammation markers and cardiovascular disease risk in} youth

Atherosclerosis is now widely accepted as a chronic inflammatory disease that is initiated by vascular injury induced by oxidised LDL, reactive oxygen species, infection, etc. ${ }^{1,2}$. A key feature in the inflammation hypothesis is the recognition that circulating immune cells are recruited to the inflamed vessel through the interaction between adhesion molecules and chemokines. We cannot study in vivo inflammatory processes in vessels of large groups of healthy young volunteers, but by analysing their blood, we can assess immune cells and their functionality, receptors and inflammatory proteins relatively easily.

Ultrasonography is used in research for the noninvasive detection of atherosclerotic changes in the arterial wall. With this method, serum C-reactive protein (CRP) concentration has been found to be associated with early arterial changes in children and adolescents ${ }^{3}$, supporting the hypothesis that inflammation plays a role in the pathogenesis of early atherosclerosis.

CRP concentrations are easily, accurately and fairly inexpensively measured in blood. It is an acute phase reactant and a very sensitive marker of inflammation. High CRP levels have no specificity in differentiating disease entities from one another, but despite its lack of specificity, CRP has now emerged as one of the most powerful predictors of cardiovascular risk. In a direct comparison of a panel of inflammatory and lipid markers in predicting cardiovascular events in adults, CRP could surpass other classical risk markers, including LDL cholesterol ${ }^{4,5}$. The American Heart Association and the Center for Disease Control and Prevention in the USA issued a joint statement confirming that 
CRP is the best and most clinically useful of the markers of inflammation currently available, with the following cut-off points for assessing CVD risk: low risk (CRP $<1.0 \mathrm{mg} / \mathrm{l}$ ), average risk $(1.0-3.0 \mathrm{mg} / \mathrm{l})$, and high risk $(>3.0 \mathrm{mg} / \mathrm{l})^{6}$. However, these cut-off points are for adults and no reference ranges are yet defined for children or adolescents. Using data from 524 Spanish adolescents, 13-18 years of age, from the multicentre "AVENA" study as an example to classify apparently healthy adolescents with these cut-offs, $68 \%$ of the subjects were at low risk, $23 \%$ at average risk and $7 \%$ at high risk. Concentrations ranged from 0.04 to $47.37 \mathrm{mg} / \mathrm{l}$ and the median was $0.64 \mathrm{mg} / 1 ; 2 \%$ of the subjects had CRP levels $>10 \mathrm{mg} / \mathrm{l}^{7}$. These last high CRP concentrations most probably reflected an acute phase response to infectious disease or immune disorders characterised by acute inflammation, a process which is natural and necessary to combat any infection. In research of chronic inflammation however, to minimise the confounder of an ongoing infection, subjects with values $>10 \mathrm{mg} / \mathrm{l}$ should be excluded from analyses or studied on another occasion.

The main way for the immune cells to communicate with each other and with other cells is regulated by small proteins called cytokines. They are produced mainly by cells within the immune system, e.g. monocytes, macrophages, and $\mathrm{T}$ lymphocytes, but also by other cells, e.g. mast cells, fibroblasts, endothelial cells, neurons and also by adipocytes. Cytokines interact in a complicated way, inducing or inhibiting the production and the effects of each other. Sometimes, especially in inflammation, cytokines appear in the circulation most often in order to regulate the gene expression in a specific organ or cell type. This is the case when IL-1, IL-6 and TNF- $\alpha$ induce the hepatic synthesis of acute phase proteins.

Other markers are present in chronic inflammation that may have pathophysiological roles in the development of chronic diseases, including adhesion molecules and acute phase proteins other than CRP, such as complement factors, but these markers are only just beginning to be included in population based studies of healthy asymptomatic young people.

Throughout this review we suggest that chronic low-grade inflammation could promote future disease. It is, however, important to stress that the serum values of inflammatory proteins presented in the discussed epidemiological studies are always within normal ranges, which implies that the populations are healthy and that the meaning of chronically high values may be clinically significant in the long term.

\section{Obesity-induced chronic low-grade inflammation}

Obesity has repeatedly been associated with moderately raised levels of inflammation in adults, and this observation has led to the view that obesity is characterised by a state of chronic low-grade inflammation ${ }^{8}$. There is also increasing evidence to show that chronic subclinical inflammation is associated with the metabolic dysfunction associated with obesity, which links causally to insulin resistance and the metabolic syndrome ${ }^{9}$. Even in children and adolescents, metabolic complications of excess body fat are detectable, and in clinical settings the state of chronic inflammation in these subjects is, too, well established $^{10-13}$.
Several large-scale population based studies demonstrating that proxies for body fat (i.e. BMI and skinfold thickness) are significant predictors of CRP also in young apparently healthy children and adolescents from around the world have been published in the last 5 years ${ }^{14-16}$. Pro-inflammatory cytokines, such as IL- 6 and TNF- $\alpha$ have also been studied in overweight paediatric populations, and while IL-6 has shown good correlations with body fat ${ }^{11,17}$, there is no consensus regarding TNF- $\alpha$ concentrations; some studies have found elevated concentrations ${ }^{17,18}$, while others have not ${ }^{11,19}$.

Other inflammatory markers are less well studied. The Spanish adolescent population has a high prevalence of overweight, including central obesity ${ }^{20}$. In this population (data from the AVENA study), associations between proxies of body fat and complement factors (i.e. $\mathrm{C} 3$ and $\mathrm{C} 4$ ) were generally stronger than for CRP, and C3 was also shown to be associated with abdominal obesity measured by waist circumference, independently of total body fat ${ }^{21}$. In this same study, ceruloplasmin correlated with body fat, but only in girls ${ }^{21}$. There could be several causes for the association between overweight and body fat with chronic low-grade inflammation, but the mechanisms are yet not entirely understood. Adipose tissue is known to have endocrine functions. The most direct mechanism could be that adipose tissue is a direct source of pro-inflammatory cytokines such as IL-6 and TNF- $\alpha$, which in turn induce hepatic synthesis of acute-phase proteins (i.e. CRP and complement factors) ${ }^{2,9}$. An important question still to be answered is how adiposity regulates inflammation during childhood and adolescent overweight and obesity, in order to increase our knowledge of the possible mechanisms involved in the development of related diseases or disorders.

\section{Anti-inflammatory effects of physical activity and fitness?}

In disease-free children and adolescents, several interesting studies have assessed the interaction between inflammation, physical activity, cardiorespiratory fitness and fatness $7,17,22-28$. Physical activity is defined as any body movement produced by skeletal muscular action that increases energy expenditure. Exercise is a sub-category of physical activity which is planned, structured and repetetive with the objective of increasing one or more components of physical fitness, often cardiorespiratory fitness. Cardiorespiratory fitness is the overall capacity of the cardiovascular and respiratory systems and the ability to carry out prolonged strenuous exercise ${ }^{29}$.

Leisure time exercise in children (assessed by questionnaire) has been found to have negative correlations with serum IL-6 concentrations, independently of adiposity and fat localisation ${ }^{24}$, and in 10 year-old children, a borderline significant negative association was observed between CRP and self-reported physical activity, independently of ponderal inde $^{22}$. Only one study has used accelerometry (an objective measure of total physical activity, compared to leisure time physical activity or exercise) instead of questionnaires. In this study of 9 year-old children from the Swedish part of the European Youth Heart Study, no associations with physical activity and either CRP, fibrinogen, C3 or C4 were observed $^{27}$.

It has been estimated that as many as $20 \%$ of Spanish adolescents have a level of cardiorespiratory fitness that could 
be indicative of future $\mathrm{CVD}^{30}$, and data from this study (the AVENA study) showed that cardiorespiratory fitness seemed to counteract the relationship between obesity and increased cardiovascular risk in these adolescents ${ }^{31,32}$. In regard to inflammatory markers, we showed that fitness was independently associated with C3 (but not to CRP, C4 or ceruloplasmin) after adjusting for BMI in male adolescents, which might indicate, at least in part, an anti-inflammatory effect, independent of adiposity, of fitness in this population ${ }^{7}$. Several other studies in children, adolescents and young adults have correlated fitness with lower levels of CRP, IL-6 and $\mathrm{TNF}-\alpha^{22,26-28}$ but this correlation has not always been clearly independent of body fat.

Because low physical activity and fitness, and increased adiposity, often occur in combination, masking their independent effects, more research is needed to elucidate this complex relationship.

\section{Diet and inflammation}

Many interesting reviews in this supplement address the interactions between nutrients and immune status, including inflammation, in animals or in adults, and most studies are concerned with diseased populations or ones with a high risk of a certain disease. Studies in healthy adults are scarce and only in recent years have a few epidemiological studies associated dietary patterns or habits with biomarkers of inflammation. This research question is addressed even less frequently in large-scale populations of healthy adolescents.

In one study of children and adolescents associating food intake with inflammation, total fat intake and percentage of energy from fat was shown to predict CRP levels in 6 to $14 \mathrm{y}$ old Swiss children, independent of $\mathrm{BMI}^{11}$. In this same study, meat consumption predicted serum IL-6 levels but intakes of vitamin $\mathrm{C}, \mathrm{E}$ and $\beta$-carotene predicted neither CRP nor IL-6 concentrations ${ }^{11}$. In the NHANES III cohort, magnesium consumption has very recently been associated with CRP levels, showing that subjects with intakes below the Recommended Daily Allowances (RDA) are more likely to have elevated CRP levels ${ }^{33}$. It has been reported repeatedly that low antioxidant vitamin concentrations and a reduced antioxidant capacity are common in obese children ${ }^{34,35}$. Overweight adolescents with elevated CRP and IL- 6 concentrations have also been shown to have higher plasma concentrations of saturated fatty acids and lower concentrations of n-3 polyunsaturated fatty acids ${ }^{36}$. Serum levels of vitamins or fatty acids are just surrogates of food intake, which is reflected in the fact that in NHANES III, obese children and adolescents (619 y) had lower serum $\alpha$-tocopherol and $\beta$-carotene, but no significant differences in intake (assessed by $24 \mathrm{~h}$ recalls and food-frequency questionnaires) of these vitamins, or intakes of fruits or vegetables, could be observed ${ }^{37}$.

In adults, the Nurse's Health Study carried out in women found a dietary pattern strongly related to inflammatory markers was characterised by sugar-sweetened soft drinks, refined grains, diet soft drinks, and processed meat but low intakes of wine, coffee, cruciferous vegetables and yellow vegetables ${ }^{38}$. This dietary pattern also raised the risk of the development of type 2 diabetes in a follow-up study of the same women (Nurses' Health Study II). This finding may provide evidence that the association between dietary factors and the risk of type 2 diabetes may be mediated in part by inflammation. The Mediterranean diet (characterised as rich in olive oil, fruits, nuts, vegetables, wholegrain breads, fish and lean meat) has also been studied together with inflammatory markers in epidemiological studies in adults, associating those who most strictly followed the Mediterranean diet with lower CRP and IL-6 levels than those who did not follow this type of diet ${ }^{39}$.

The difficulties with assessing food intake in humans, and especially in young people are well known ${ }^{40}$, and ensuring adequate statistical power when examining the relationship between dietary intakes and inflammatory markers in healthy populations is a difficult task. This challenge could explain some of the lack of published information, but should certainly not be a deterrent to pursuing this line of research.

\section{Conclusions}

More and more evidence suggest that low-grade chronic inflammation predicts an increased risk of chronic disease and promotes its progression. Both the underlying causes and preventive measures for this state in early ages are related to lifestyle factors. The association between diet, physical activity and obesity is well established, and between obesity and inflammation (Fig. 1). There may, however, also be indirect associations between dietary compounds, patterns of physical activity and fitness with inflammation. For public health strategies and preventive purposes, it is of importance to understand the relative influence of modifiable factors on low-grade inflammation from an early age. Understanding the interactions between nutrition, appropriate weight and physical activity and physical fitness, may be of importance in reducing the future cardiovascular disease risk of young people.

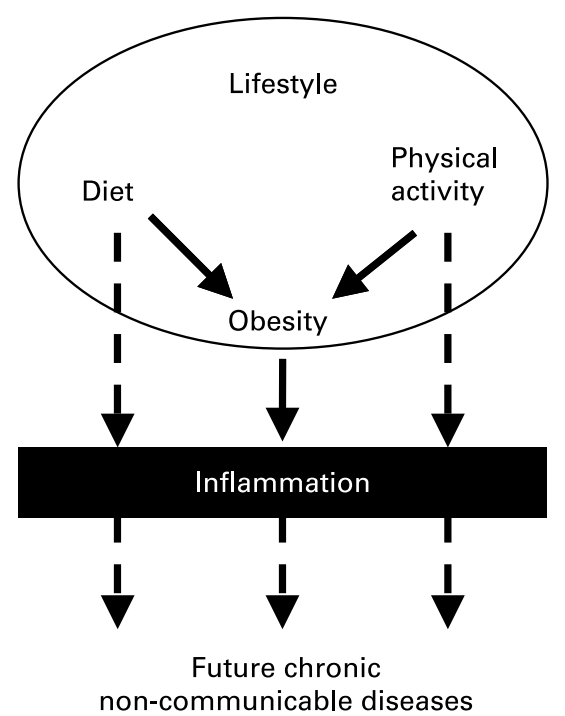

Fig. 1. Consequences of an obesogenic lifestyle on the development of chronic diseases through inflammation. Shown in the figure is the hypothesis that lifestyle predicts future chronic diseases indirectly through inflammation. In apparently healthy adolescents, the association between diet or physical activity and obesity is well established, and between obesity and inflammation as well. There may also be indirect associations between dietary compounds and patterns or physical activity and fitness with inflammation. 


\section{Conflict of interest statement}

JW, EN, JR, LAM, MS and AM have no conflicts of interest to declare. AM was the principal investigator of the AVENA study. This review was drafted by JW and has partly been published in her doctoral thesis ${ }^{7}$, with MS, AM, LAM and EN as tutors. All authors discussed and approved the final version.

\section{References}

1. Ross R (1999) Atherosclerosis is an inflammatory disease. Am Heart J 138, S419-S420.

2. Hansson GK (2005) Inflammation, atherosclerosis, and coronary artery disease. $N$ Engl J Med 352, 1685-1695.

3. Jarvisalo MJ, Harmoinen A, Hakanen M, Paakkunainen U, Viikari J, Hartiala J, Lehtimaki T, Simell O \& Raitakari OT (2002) Elevated serum C-reactive protein levels and early arterial changes in healthy children. Arterioscler Thromb Vasc Biol 22, 1323-1328.

4. Ridker PM, Buring JE, Cook NR \& Rifai N (2003) C-reactive protein, the metabolic syndrome, and risk of incident cardiovascular events: an 8-year follow-up of 14719 initially healthy American women. Circulation 107, 391-397.

5. Ridker PM, Stampfer MJ \& Rifai N (2001) Novel risk factors for systemic atherosclerosis: a comparison of C-reactive protein, fibrinogen, homocysteine, lipoprotein(a), and standard cholesterol screening as predictors of peripheral arterial disease. JAMA 285, 2481-2485.

6. Pearson TA, Mensah GA, Alexander RW, et al. (2003) Markers of inflammation and cardiovascular disease: application to clinical and public health practice: A statement for healthcare professionals from the Centers for Disease Control and Prevention and the American Heart Association. Circulation 107, 499-511.

7. Warnberg J (2006) Inflammatory status in adolescents; the impact of health determinants such as overweight and fitness. $\mathrm{PhD}$ Thesis, (University Press, Stockholm).

8. Trayhurn P \& Wood IS (2004) Adipokines: inflammation and the pleiotropic role of white adipose tissue. Br J Nutr 92, 347-355.

9. Hotamisligil GS (2006) Inflammation and metabolic disorders. Nature 444, 860-867.

10. Weiss R \& Caprio S (2005) The metabolic consequences of childhood obesity. Best Pract Res Clin Endocrinol Metab 19, 405-419.

11. Aeberli I, Molinari L, Spinas G, Lehmann R, l'Allemand D \& Zimmermann MB (2006) Dietary intakes of fat and antioxidant vitamins are predictors of subclinical inflammation in overweight Swiss children. Am J Clin Nutr 84, 748-755.

12. Visser M, Bouter LM, McQuillan GM, Wener MH \& Harris TB (2001) Low-grade systemic inflammation in overweight children. Pediatrics 107, E13.

13. Winer JC, Zern TL, Taksali SE, et al. (2006) Adiponectin in childhood and adolescent obesity and its association with inflammatory markers and components of the metabolic syndrome. J Clin Endocrinol Metab 91, 4415-4423.

14. Warnberg J, Moreno LA, Mesana MI \& Marcos A (2004) Inflammatory mediators in overweight and obese Spanish adolescents. The AVENA Study. Int J Obes Relat Metab Disord 28, Suppl 3, S59-S63.

15. Ford ES, Galuska DA, Gillespie C, Will JC, Giles WH \& Dietz WH (2001) C-reactive protein and body mass index in children: findings from the Third National Health and Nutrition Examination Survey, 1988-1994. J Pediatr 138, 486-492.
16. Wu DM, Chu NF, Shen MH \& Wang SC (2006) Obesity, plasma high sensitivity C-reactive protein levels and insulin resistance status among school children in Taiwan. Clin Biochem 39, 810-815.

17. Halle M, Korsten-Reck U, Wolfarth B \& Berg A (2004) Lowgrade systemic inflammation in overweight children: impact of physical fitness. Exerc Immunol Rev 10, 66-74.

18. Reinehr T, Stoffel-Wagner B, Roth CL \& Andler W (2005) High-sensitive C-reactive protein, tumor necrosis factor alpha, and cardiovascular risk factors before and after weight loss in obese children. Metabolism 54, 1155-1161.

19. Gupta A, Ten S \& Anhalt H (2005) Serum levels of soluble tumor necrosis factor-alpha receptor 2 are linked to insulin resistance and glucose intolerance in children. $J$ Pediatr Endocrinol Metab 18, 75-82.

20. Moreno LA, Mesana MI, Gonzalez-Gross M, et al. (2007) Body fat distribution reference standards in Spanish adolescents: the AVENA Study. Int J Obes (Lond) Jul 3, [Epub ahead of print].

21. Warnberg J, Nova E, Moreno LA, et al. (2006) Inflammatory proteins are related to total and abdominal adiposity in a healthy adolescent population: the AVENA Study. Am J Clin Nutr 84, $505-512$.

22. Cook DG, Mendall MA, Whincup PH, Carey IM, Ballam L, Morris JE, Miller GJ \& Strachan DP (2000) C-reactive protein concentration in children: relationship to adiposity and other cardiovascular risk factors. Atherosclerosis 149, $139-150$.

23. Nemet D, Wang P, Funahashi T, Matsuzawa Y, Tanaka S, Engelman L \& Cooper DM (2003) Adipocytokines, body composition, and fitness in children. Pediatr Res 53, 148-152.

24. Platat C, Wagner A, Klumpp T, Schweitzer B \& Simon C (2006) Relationships of physical activity with metabolic syndrome features and low-grade inflammation in adolescents. Diabetologia 49, 2078-2085.

25. Isasi CR, Deckelbaum RJ, Tracy RP, Starc TJ, Berglund L \& Shea S (2003) Physical fitness and C-reactive protein level in children and young adults: the Columbia University BioMarkers Study. Pediatrics 111, 332-338.

26. Kelly AS, Wetzsteon RJ, Kaiser DR, Steinberger J, Bank AJ \& Dengel DR (2004) Inflammation, insulin, and endothelial function in overweight children and adolescents: the role of exercise. J Pediatr 145, 731-736.

27. Ruiz JR, Ortega FB, Warnberg J \& Sjostrom M (2007) Associations of low-grade inflammation with physical activity, fitness and fatness in prepubertal children; the European Youth Heart Study. Int J Obes (Lond) Jul, 24, [Epub ahead of print].

28. Williams MJ, Milne BJ, Hancox RJ \& Poulton R (2005) C-reactive protein and cardiorespiratory fitness in young adults. Eur $J$ Cardiovasc Prev Rehabil 12, 216-220.

29. Caspersen CJ, Powell KE \& Christenson GM (1985) Physical activity, exercise, and physical fitness: definitions and distinctions for health-related research. Public Health Rep 100, $126-131$.

30. Ortega FB, Ruiz JR, Castillo MJ, Moreno LA, GonzalezGross M, Warnberg J \& Gutierrez A (2005) [Low level of physical fitness in Spanish adolescents. Relevance for future cardiovascular health (AVENA study)]. Rev Esp Cardiol 58, 898-909.

31. Ortega FB, Tresaco B, Ruiz JR, et al. (2007) Cardiorespiratory fitness and sedentary activities are associated with adiposity in adolescents. Obesity (Silver Spring) 15, 1589-1599.

32. Mesa JL, Ruiz JR, Ortega FB, Warnberg J, Gonzalez-Lamuno D, Moreno LA, Gutierrez A \& Castillo MJ (2006) Aerobic physical fitness in relation to blood lipids and fasting glycaemia in adolescents: influence of weight status. Nutr Metab Cardiovasc Dis 16, 285-293. 
33. King DE, Mainous AG III, Geesey ME \& Ellis T (2007) Magnesium intake and serum C-reactive protein levels in children. Magnes Res 20, 32-36.

34. Decsi T, Molnar D \& Koletzko B (1997) Reduced plasma concentrations of alpha-tocopherol and beta-carotene in obese boys. J Pediatr 130, 653-655.

35. Molnar D, Decsi T \& Koletzko B (2004) Reduced antioxidant status in obese children with multimetabolic syndrome. Int $J$ Obes Relat Metab Disord 28, 1197-1202.

36. Klein-Platat C, Drai J, Oujaa M, Schlienger JL \& Simon C (2005) Plasma fatty acid composition is associated with the metabolic syndrome and low-grade inflammation in overweight adolescents. Am J Clin Nutr 82, 1178-1184.

37. Strauss RS (1999) Comparison of serum concentrations of alphatocopherol and beta-carotene in a cross-sectional sample of obese and nonobese children (NHANES III). National Health and Nutrition Examination Survey. J Pediatr 134, 160-165.

38. Schulze MB, Hoffmann K, Manson JE, Willett WC, Meigs JB, Weikert C, Heidemann C, Colditz GA \& Hu FB (2005) Dietary pattern, inflammation, and incidence of type 2 diabetes in women. Am J Clin Nutr 82, 675-684, quiz 714-675.

39. Chrysohoou C, Panagiotakos DB, Pitsavos C, Das UN \& Stefanadis C (2004) Adherence to the Mediterranean diet attenuates inflammation and coagulation process in healthy adults: The ATTICA Study. J Am Coll Cardiol 44, 152-158.

40. Moreno L, Gonzalez-Gross M, Kersting M, et al. (2007) Assessing, understanding and modifying nutritional status, eating habits and physical activity in European adolescents: The HELENA (Healthy Lifestyle in Europe by Nutrition in Adolescence) Study. Public Health Nutr [Epub ahead of print] 